\title{
Predicting conformational entropy of bond vectors in proteins by networks of coupled rotators
}

\author{
Anne Dhulesia, ${ }^{1, a)}$ Geoffrey Bodenhausen, ${ }^{1,2}$ and Daniel Abergel ${ }^{1, b)}$ \\ ${ }^{1}$ Départment de Chimie, Associé au CNRS, Ecole Normale Supérieure, 24, rue Lhomond, \\ 75005 Paris, France \\ ${ }^{2}$ Institut des Sciences et Ingéniérie Chimiques, Ecole Polytechnique Fédérale de Lausanne, \\ BCH 1015 Lausanne, Switzerland
}

(Received 21 April 2008; accepted 21 July 2008; published online 5 September 2008)

In this article, a formal expression for the conformational entropy of a bond vector in a protein is derived using the networks of coupled rotators model for the description of internal dynamics. Analytical relationships between NMR order parameters and conformational entropies are derived, and the possibility to extract the latter from NMR experiments is discussed. These results are illustrated in the case of the calcium-binding protein calbindin. (C) 2008 American Institute of Physics. [DOI: 10.1063/1.2969809]

\section{INTRODUCTION}

It has long been assumed that the function of a protein is embodied in its three dimensional structure. This simple picture tends to be gradually replaced by a more dynamic view according to which internal motions represent an essential ingredient of protein function, ${ }^{1}$ so that a protein "structure" should be rather viewed as a superposition of interconverting conformations. The time scales of the dynamical processes that occur in proteins span several orders of magnitude. Motions with characteristic time constants longer than microseconds can be related to important biological processes, such as enzymatic catalysis, ligand binding, and protein-protein and protein-nucleic acid interactions. Alternatively, fast internal motions (on a subnanosecond time scale) corresponding to rotations, vibrations, and librations of chemical bonds can be related to conformational entropy. Nuclear magnetic resonance (NMR) spectroscopy represents a unique tool to study protein dynamics, as it allows one to probe events on the pico- to millisecond time scale, on a residue-per-residue basis, via the measurement of spin relaxation rates. ${ }^{1-3}$ One of the most challenging issues in the study of protein dynamics is therefore to relate NMR relaxation rates to a quantitative description of internal motions. This comprises two different aspects, kinetic and equilibrium, and includes the prediction of relevant correlation functions and conformational entropies. Because internal dynamics are constrained by a potential, the internal correlation functions that are relevant to spin relaxation tend to a nonvanishing limit at long times, known in the NMR community as the squared order parameter and denoted by $S^{2}$, which is a measure of the motional amplitude of the bond vectors. ${ }^{4}$ Establishing a formal relationship between site-specific order parameters $S^{2}$ determined from experimental NMR relaxation measurements and conformational entropies of individual bond vectors is a challenging

\footnotetext{
${ }^{a)}$ Present address: Department of Chemistry, University of Cambridge, Lensfield Road, CB2 1EW, Cambridge, UK.

b) Author to whom correspondence should be addressed. Electronic mail: daniel.abergel@ens.fr.
}

task. Most approaches are based on the assumption that all vectors are independent, ${ }^{5,6}$ which affords in this case a simple link between conformational entropies of the individual vectors and of the whole protein, but may seem rather unrealistic. We have therefore investigated the possibility of using networks of coupled rotators ${ }^{7}$ (NCRs) to predict conformational entropies and derive their relationships with the order parameters.

\section{RESULTS}

As detailed elsewhere, ${ }^{7}$ in the NCR approach the protein is represented by an ensemble of vectors that are parallel to chemical bonds and assumed to undergo wobbling motions about their average direction. Each pair of network vectors $\left(\mathbf{u}_{i}, \mathbf{u}_{j}\right)$ is coupled by a potential $U_{i j}$ that tends to restore the instantaneous angle $\theta_{i j}$ between them to its equilibrium value $\theta_{0 i j}$, given by the three dimensional protein structure determined by X ray or NMR. These pair potentials $U_{i j}$ depend on local atomic densities $\rho_{i}$ and $\rho_{j}$, as well as on a scaling constant $\kappa_{0}$, common to all vectors in the protein, ${ }^{7-9}$

$$
U_{i j}=-\rho_{i} \rho_{j} \kappa_{0} k T P_{2}\left(\cos \left(\theta_{i j}-\theta_{0 i j}\right)\right),
$$

where $P_{2}(x)$ is the second Legendre polynomial. Because proteins are densely packed molecules, the bond vector motions are assumed to be restricted. The potentials $U_{i j}$ can then be expanded up to the second order in terms of the components $\left(X_{i}, Y_{i}\right)$ and $\left(X_{j}, Y_{j}\right)$ of $\mathbf{u}_{i}$ and $\mathbf{u}_{j}$ in their respective local reference frames where the $z$ axes are parallel to the average bond vectors $\left\langle\mathbf{u}_{i}\right\rangle$ and $\left\langle\mathbf{u}_{j}\right\rangle$. In the NCR model, the coupled vectors diffuse in the potential $U=\Sigma_{i<j} U_{i j}$ and are driven by stochastic Langevin forces. The dynamics are described by rotational Langevin equations, ${ }^{10}$ which, due to the above approximations, allow one to determine the evolution of various averages of the components of the vectors $\mathbf{u}_{i}{ }^{7}$ These can then be used to predict NMR observables. Of central importance in what follows, the order parameter $S_{i}^{2}$, defined as the limit of the internal autocorrelation function $C_{i}^{1}(t)$ of the bond vector $\mathbf{u}_{i}$, can be expressed as. ${ }^{7,11}$ 


$$
S_{i}^{2}=\lim _{t \rightarrow \infty} C_{i}^{I}(t)=1-3\left(\left\langle X_{i}^{2}\right\rangle+\left\langle Y_{i}^{2}\right\rangle\right)
$$

More generally, as only the second moments of the components $X_{i}, Y_{i}$ of the coupled vectors are nonzero, ${ }^{7}$ the equilibrium statistical information is entirely described by the covariance matrix $\mathbf{M}$,

$$
\mathbf{M}=\left(\begin{array}{ccccc}
\left\langle X_{1}^{2}\right\rangle & \left\langle X_{1} Y_{1}\right\rangle & \ldots & \left\langle X_{1} X_{N}\right\rangle & \left\langle X_{1} Y_{N}\right\rangle \\
\left\langle X_{1} Y_{1}\right\rangle & \left\langle Y_{1}^{2}\right\rangle & \ldots & \left\langle Y_{1} X_{N}\right\rangle & \left\langle Y_{1} Y_{N}\right\rangle \\
\vdots & \vdots & \vdots & \vdots & \vdots \\
\left\langle Y_{N} X_{1}\right\rangle & \left\langle Y_{N} Y_{1}\right\rangle & \ldots & \left\langle Y_{N} X_{N}\right\rangle & \left\langle Y_{N}^{2}\right\rangle
\end{array}\right) .
$$

In this case, the probability distribution function $p(\mathbf{X})$ of the variable $\mathbf{X}=\left[X_{1}, Y_{1}, \ldots, X_{N}, Y_{N}\right]^{t}$ is Gaussian, ${ }^{12}$

$$
p(\mathbf{X})=\frac{\sqrt{\operatorname{det} \mathbf{H}}}{(2 \pi)^{N}} e^{-\mathbf{X}^{t} \mathbf{H} \mathbf{X} / 2},
$$

where $\mathbf{H}=\mathbf{M}^{-1}$ is the Hessian matrix. Thus, Eq. (4) corresponds to a Boltzmann distribution with the average potential $\mathbf{X}^{t} \mathbf{H X} / 2$ and the partition function $\mathcal{Z}=(2 \pi)^{N} / \sqrt{\operatorname{det} \mathbf{H}}$.

We shall denote by $S_{i \mathrm{NCR}}^{e}$ the conformational entropy associated with the vector $\mathbf{u}_{i}$, the motion of which is described by the variables $\left(X_{i}, Y_{i}\right)$. By definition, $S_{i \mathrm{NCR}}^{e}$ is obtained from the probability distribution $p\left(X_{i}, Y_{i}\right)$ of the components $\left(X_{i}, Y_{i}\right)$,

$$
S_{i \mathrm{NCR}}^{e}=-k \int p\left(X_{i}, Y_{i}\right) \log p\left(X_{i}, Y_{i}\right) d X_{i} d Y_{i},
$$

where the marginal distribution function $p\left(X_{i}, Y_{i}\right)$ is obtained by integrating $p(X)$ over all variables other than $X_{i}$ and $Y_{i}$. For simplicity, we consider the first vector, $i=1$,

$$
\begin{aligned}
& p\left(X_{1}, Y_{1}\right)=\int p(X, Y) d X^{N-1} d Y^{N-1}, \\
& p\left(X_{1}, Y_{1}\right)=\frac{\sqrt{\operatorname{det} \mathbf{H}}}{(2 \pi)^{N}} \int d X^{N-1} d Y^{N-1} e^{-(1 / 2) \mathbf{X}^{t} \mathbf{H X},}
\end{aligned}
$$

with the shorthand notations $d X^{N-1}=d X_{2} \cdots d X_{N}$ and $d Y^{N-1}$ $=d Y_{2} \cdots d Y_{N}$. Standard properties of partial Gaussian integrals give

$$
p\left(X_{1}, Y_{1}\right)=\frac{1}{2 \pi} \sqrt{\frac{\operatorname{det} \mathbf{H}}{\operatorname{det} W_{0}^{\left(X_{1} Y_{1}\right)}}} e^{-(1 / 2)\left[X_{1} Y_{1}\right] U_{1}^{(2)}\left[X_{1} Y_{1}\right]^{\dagger}},
$$

where the matrices $W_{0}^{\left(X_{1} Y_{1}\right)}$ and $U_{1}^{(2)}$ result from the block decomposition of $\mathbf{H}$,

$$
\mathbf{H}=\left(\begin{array}{ll}
U_{0}^{\left(X_{1} Y_{1}\right)} & V^{\left(X_{1} Y_{1}\right)} \\
V^{\left(X_{1} Y_{1}\right) t} & W_{0}^{\left(X_{1} Y_{1}\right)}
\end{array}\right),
$$

$U_{0}^{\left(X_{1} Y_{1}\right)}$ is a $2 \times 2$ matrix that connects $X_{1}$ and $Y_{1}$, and we define $U_{1}^{(2)}$ as

$$
U_{1}^{(2)}=U_{0}^{\left(X_{1} Y_{1}\right)}-V^{\left(X_{1} Y_{1}\right)}\left(W_{0}^{\left(X_{1} Y_{1}\right)}\right)^{-1} V^{\left(X_{1} Y_{1}\right) t} .
$$

Thus, $U_{1}^{(2)}$ is the effective diffusion potential of vector $\mathbf{u}_{1}$, the conformational entropy of which, $S_{1, \mathrm{NCR}}^{e}$, can be derived from Eq. (5),

$$
\begin{aligned}
-\frac{S_{1, \mathrm{NCR}}^{e}}{k}= & \log \left(\frac{\sqrt{\operatorname{det} U_{1}^{(2)}}}{2 \pi}\right)-\frac{U_{11}^{(2)}}{2}\left\langle X_{1}^{2}\right\rangle-\frac{U_{22}^{(2)}}{2}\left\langle Y_{1}^{2}\right\rangle \\
& -\frac{U_{12}^{(2)}+U_{21}^{(2)}}{2}\left\langle X_{1} Y_{1}\right\rangle,
\end{aligned}
$$

where the $U_{i j}^{(2)}$ are the matrix elements of the symmetric matrix $U_{1}^{(2)}$, with $U_{12}^{(2)}=U_{21}^{(2)}$. Obviously, the same result can be obtained for any vector of the network by changing indices 1 to $i$ in Eqs. (5)-(8) and by proper row and column permutations in Eq. (9).

Equation (11) defines the relationship between the covariance matrix and the conformational entropy $S_{1, \mathrm{NCR}}^{e}$ of the vector $\mathbf{u}_{1}$ in the framework of the NCR model. Therefore, when amide $\mathrm{NH}$ vectors are included in the network, which is required to predict ${ }^{15} \mathrm{~N}$ relaxation rates, the NCR model affords a means of predicting local conformational entropy, based on the sole knowledge of the molecular structure. The averages $\left\langle X_{1}^{2}\right\rangle,\left\langle Y_{1}^{2}\right\rangle$, and $\left\langle X_{1} Y_{1}\right\rangle$ that appear in Eq. (11) can be expressed in terms of the matrix elements of the effective potential $U_{1}^{(2)}$,

$$
\begin{aligned}
& \left\langle X_{1}^{2}\right\rangle=\frac{U_{22}^{(2)}}{\operatorname{det} U_{1}^{(2)}}, \\
& \left\langle Y_{1}^{2}\right\rangle=\frac{U_{11}^{(2)}}{\operatorname{det} U_{1}^{(2)}}, \\
& \left\langle X_{1} Y_{1}\right\rangle=-\frac{\sqrt{\operatorname{det} U_{1}^{(2)}}\left(U_{12}^{(2)}+U_{21}^{(2)}\right)}{\left(U_{22}^{(2)} U_{11}^{(2)}-\left(U_{12}^{(2)}+U_{21}^{(2)}\right)^{2}\right)^{3 / 2}} .
\end{aligned}
$$

These relations can be simplified by going into the frame of the eigenvectors of $U_{1}^{(2)}$, leading to a new simpler formulation of the conformational entropy of the bond vector,

$$
-\frac{S_{1, \mathrm{NCR}}^{e}}{k}=\log \left(\frac{\sqrt{\operatorname{det} U_{1}^{(2)}}}{2 \pi}\right)-1 .
$$

The connection between Lipari-Szabo order parameters $S^{2}$, as obtained from NMR relaxation experiments ${ }^{4,13}$ or from residual dipolar couplings and conformational entropy associated with a $\mathrm{NH}$ bond vector has been the subject of many studies aiming at extracting thermodynamic quantities from NMR experiments. ${ }^{1,5,14-16}$ Since both order parameters and conformational entropies can be predicted by the NCR model, the relationship between them can be easily investigated. Thus, Eqs. (2), (11), and (13) determine the formal connection between $S_{1, \mathrm{NCR}}^{2}$ and the conformational entropy $S_{1, \mathrm{NCR}}^{e}$ associated with NH vectors.

To illustrate this point, we applied these formulas to the case of the protein calbindin. ${ }^{17}$ In all what follows, we used a NCR comprising all backbone $\mathrm{NH}$ and $\mathrm{CO}$ bond vectors that are within $7.5 \AA$ of each other. ${ }^{18}$ The constant $\kappa_{0}$ is fixed to 3 for all pairwise potentials and the atomic density $\rho_{i}$ for $\mathrm{NH}$ or $\mathrm{CO}$ bond vectors is determined by the number of atoms within $7.5 \AA$ of the $\mathrm{C}$ and $\mathrm{N}$ atoms, respectively. The equilibrium values of the internuclear angles $\theta_{0 i j}$ are taken 

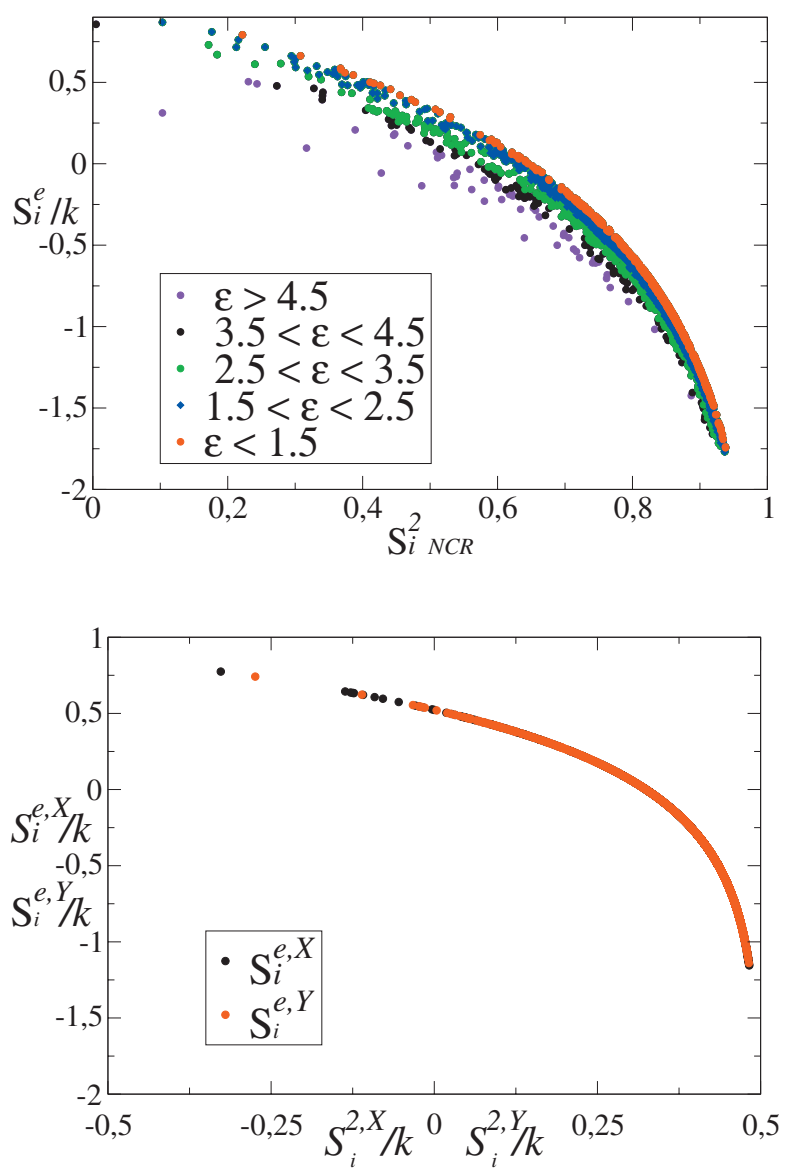

FIG. 1. (Color) Top: Plot of the conformational entropy $S_{i, \mathrm{NCR}}^{e}$ vs $S_{i i}^{2} \mathrm{NCR}$ color coded as a function of the anisotropy $\epsilon$ of the effective potential (see text for the definition of $\epsilon$ ). Bottom: "Directional" entropies $S_{i}^{e, Y^{\prime}}$ and $S_{i}^{e, X^{\prime}}$ of amide $\mathrm{NH}$ bond vectors.

from the 33 (PDB entry 1CLB) and 10 (PBD entry 1B1G) NMR structures of the free and calcium bound states of calbindin, respectively: ${ }^{17,19}$ the dispersion among the structures within these ensembles leads to standard deviations in all quantities computed by the NCR model. As shown previously, this set of parameters permits the calculation of order parameters $S_{i, \mathrm{NCR}}^{2}$ according to Eq. (2), with linear correlation coefficients relative to experimental data of $0.74 \pm 0.08$ and $0.71 \pm 0.10$ for the free and bound states, respectively. ${ }^{9} \mathrm{It}$ also allows one to determine the covariance matrix $M$ [Eq. (3)], which contains all the necessary information to compute the conformational entropy of the bond vectors, according to Eqs. (4)-(13). We will only present results relative to $\mathrm{NH}$ bond vectors, as no NMR experimental order parameters $S^{2}$ are currently available for $\mathrm{CO}$ bonds of calbindin to validate our calculated values. However, the same conclusion regarding the link between bond vector conformational entropy $S_{i, \mathrm{NCR}}^{e}$ and order parameter $S_{i, \mathrm{NCR}}^{2}$ holds for $\mathrm{CO}$ and $\mathrm{NH}$ bond vectors (data not shown), as it would for any type of bond vectors in a protein. The plot of $S_{i, \mathrm{NCR}}^{e}$ versus $S_{i, \mathrm{NCR}}^{2}$ is depicted in Fig. 1, where the scattered graph on the top shows a monotonously decaying trend. This is in accordance with the expectation that conformational entropy decreases as the order parameter increases toward its rigid limit $S_{1, \mathrm{NCR}}^{2}=1$. However, in contrast to most earlier models, ${ }^{5,15,16}$ no one-toone correspondence exists between the two quantities. The graph reflects the fact that vectors with the same order parameter $S_{i, \mathrm{NCR}}^{2}$ may have different conformational entropies, and conversely, vectors that have the same $S_{i, \mathrm{NCR}}^{e}$ are not necessarily associated with the same order parameter. Obviously, neither quantity provides a complete characterization of the local disorder.

Alternatively, $S_{i, \mathrm{NCR}}^{e}$ can be split into contributions along the two orthogonal directions of the eigenvectors of $U_{i}^{(2)}, X_{1}^{\prime}$, and $Y_{1}^{\prime}$,

$$
S_{i}^{e, X^{\prime}}=-k \int p\left(X_{i}^{\prime}\right) \log p\left(X_{i}^{\prime}\right)=\frac{k}{2}\left[\log \left(2 \pi\left\langle\mathbf{X}_{i}^{\prime 2}\right\rangle\right)+1\right],
$$

$$
S_{i}^{e, Y^{\prime}}=-k \int p\left(Y_{i}^{\prime}\right) \log p\left(Y_{i}^{\prime}\right)=\frac{k}{2}\left[\log \left(2 \pi\left\langle\mathbf{Y}_{i}^{\prime 2}\right\rangle\right)+1\right] .
$$

Here, $p\left(X_{i}^{\prime}\right)$ and $p\left(Y_{i}^{\prime}\right)$ denote the marginal averages for the single variables $X_{i}^{\prime}$ and $Y_{i}^{\prime}$ that define the $\mathrm{N}_{i} \mathrm{H}_{i}$ vector displacements along the principal axes of the effective potential $U_{i}^{(2)}$. Equations (14) and (15) are analogous to the expression derived by Yang and Kay for a harmonic potential that depends on a single variable. ${ }^{16}$ Remarkably, it can be shown that the additivity relationship $S_{i, \mathrm{NCR}}^{e}=S_{i}^{e, \mathrm{X}^{\prime}}+S_{i}^{e, \mathrm{Y}^{\prime}}$ holds in the eigenframe of the effective potential $U_{i}^{(2)}$. Equations (14) and (15) show that, in contrast to the above results, there is a simple one-to-one relationship between conformational entropy and the pseudo-order parameter along a single direction, defined as $S_{i}^{X^{\prime} 2}=1 / 2-3\left\langle X_{i}^{\prime 2}\right\rangle$ (see Fig. 1). The order parameter $S_{i, \mathrm{NCR}}^{2}$ is therefore directly related to the sum of the conformational entropies $S_{i}^{e, X^{\prime}}$ and $S_{i}^{e, Y^{\prime}}$ of the variables $X_{i}^{\prime}$ and $Y_{i}^{\prime}$ [see Eqs. (2), (14), and (15)],

$$
S_{i, \mathrm{NCR}}^{2}=1-\frac{3}{2 \pi}\left\{\exp \left(2 \frac{S_{i}^{e, X^{\prime}}}{k}-1\right)+\exp \left(2 \frac{S_{i}^{e, Y^{\prime}}}{k}-1\right)\right\} \text {. }
$$

However, $S_{i, \mathrm{NCR}}^{2}=S_{j, \mathrm{NCR}}^{2}$ for two network vectors does not imply that $\left\langle X_{i}^{\prime 2}\right\rangle=\left\langle X_{j}^{\prime 2}\right\rangle$ and $\left\langle Y_{i}^{\prime 2}\right\rangle=\left\langle Y_{i}^{\prime 2}\right\rangle$, but only the less stringent relation $\left\langle X_{i}^{\prime 2}+Y_{i}^{\prime 2}\right\rangle=\left\langle X_{j}^{\prime 2}+Y_{j}^{\prime 2}\right\rangle$ [see Eq. (2)]. The degeneracy of the conformational entropy $S_{i}^{e}$ is embodied in the fact that the effective potential depends on two independent variables, $X_{i}$ and $Y_{i}$, and is characterized by a pair of eigenvalues and the associated pair of eigenvectors (a similar effect was observed by Yang and Kay when relating conformational entropy of individual bond vectors to a twoparameter diffusion-in-a-cone model ${ }^{16}$ ). In the NCR model, the local ellipticity of the effective potential is determined by the ratio of its largest to smallest eigenvalues and defines the local anisotropy $\epsilon$. Whereas the order parameter is a quantity that does not bear any information about the motional anisotropy in the local $\left(X_{i}, Y_{i}\right)$ plane, as attested by its invariance through a rotation about the $z$-axis of the local frame, this is not the case for $\left\langle X_{i}^{2}\right\rangle$ and $\left\langle Y_{i}^{2}\right\rangle$. Thus, two vectors have equal conformational entropies only when they have the same order parameters and when the anisotropies $\epsilon$ of their effective potentials are equal. Therefore, the variations of the local 


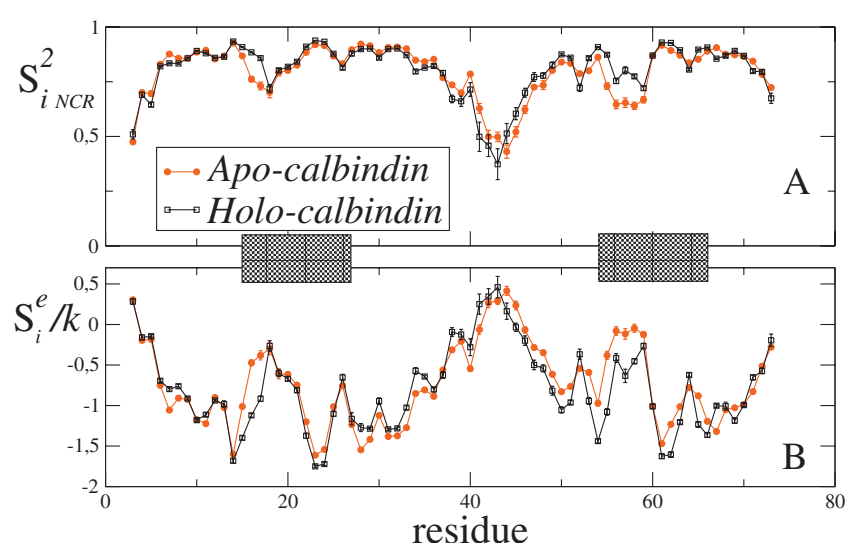

FIG. 2. (Color) Plot of the predicted order parameters $S_{i, \mathrm{NCR}}^{2}$ (top) and conformational entropy $S_{i, \mathrm{NCR}}^{e}$ (bottom) in the protein calbindin. Values for the apo- and holo-calbindin are depicted by filled circles and open squares. The calcium-binding sites (residues 14-27 and 54-65) are indicated by shaded boxes.

anisotropies $\epsilon$ from a network vector to another are responsible for the absence of a one-to-one correspondence between the bond vector conformational entropies and the order parameters. This is illustrated in Fig. 1, where the plot of $S_{i, \mathrm{NCR}}^{e}$ versus $S_{i, \mathrm{NCR}}^{2}$ is depicted with different colors for various anisotropies $\epsilon$ of the effective potential.

In general, the quest for an order parameterconformational entropy relationship is motivated by the possibility to predict thermodynamic quantities, such as $\Delta G$ or $\Delta S$, upon ligand binding or protein-protein interactions. Standard experimental techniques, such as isothermal calorimetry, allow one to determine global thermodynamic quantities, relative to the whole protein, and NMR relaxation brings the possibility of breaking down these quantities into residue contributions. Provided that the motions of the residues are decoupled, the partition function of the protein can be factorized into the product of the partition functions of individual vectors and the entropy contributions of each residue add up to the total entropy of the protein. ${ }^{5,16}$ Such a factorization becomes irrelevant to the NCR model, since the latter relies on couplings between all rotators throughout the protein. Nevertheless, as demonstrated above, since one can obtain the protein's partition function from the NCR covariance matrix, it is possible to calculate conformational entropies of individual residues, on the one hand, and the thermodynamical entropy of the protein, on the other hand. Of course, as expected, the $S_{i, \mathrm{NCR}}^{e}$ do not add up to yield the thermodynamical entropy of the protein.

We applied the NCR approach to the calculation of conformational entropies of $\mathrm{N}_{i} \mathrm{H}_{i}$ vectors $\mathbf{u}_{i}$ in the $\mathrm{Ca}^{2+}$-bound ${ }^{17}$ and $\mathrm{Ca}^{2+}$-free ${ }^{19}$ forms of calbindin. ${ }^{9}$ Results are depicted in Fig. 2(b) and compared to $S_{i, \mathrm{NCR}}^{2}$ order parameters obtained in previous work, shown in Fig. 2(a). Observation of Fig. 2 reveals that both quantities provide similar dynamical pictures for most residues. Indeed, larger $S_{i, \mathrm{NCR}}^{2}$ are associated with lower $S_{i, \mathrm{NCR}}^{e}$ and vice versa. In particular, the rigidity gained in the $\mathrm{Ca}^{2+}$ binding loops ${ }^{20}$ in holo-calbindin is reflected by an increase in $S_{i, \mathrm{NCR}}^{2}$, so that $\Delta S_{i, \mathrm{NCR}}^{2}$ $=S_{i, \mathrm{NCR}}^{2}($ holo $)-S_{i, \mathrm{NCR}}^{2}($ apo $)>0$ (as previously observed $\left.{ }^{9}\right)$ and a decrease in conformational entropy, $\Delta S_{i, \mathrm{NCR}}^{e}=S_{i, \mathrm{NCR}}^{e}($ holo $)$

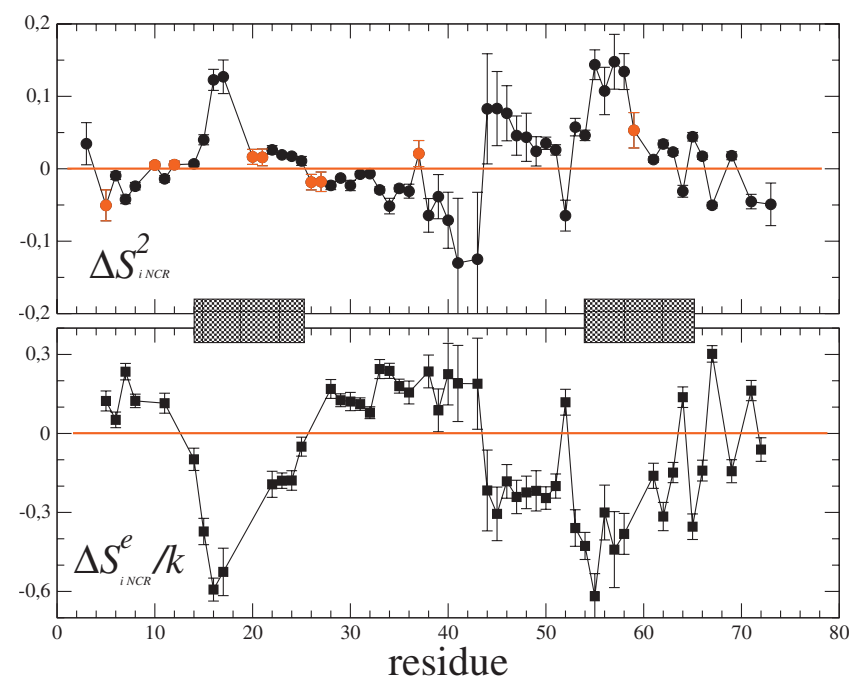

FIG. 3. (Color) Differences of $S_{i, \mathrm{NCR}}^{2}$ and $S_{i, \mathrm{NCR}}^{e}$ between apo- and holocalbindin. Only differences larger than the error bars are plotted. The red circles indicate $\mathrm{NH}$ vectors for which $\Delta S_{i, \mathrm{NCR}}^{2} \neq 0$ and $\Delta S_{i, \mathrm{NCR}}^{e} \approx 0$ (residues $5,10,12,20,21,26,27,37$, and 59).

$-S_{i, \mathrm{NCR}}^{e}($ apo $)<0$. The analysis of the differences of $S_{i, \mathrm{NCR}}^{2}$ and $S_{i, \mathrm{NCR}}^{e}$ between apo- and holo-calbindin shows that for some of the residues, $\Delta S_{i, \mathrm{NCR}}^{2} \neq 0$, whereas $\Delta S_{i, \mathrm{NCR}}^{e}=0$ (residues $5,10,12,20,21,26,27,37$, and 59) (Fig. 3).

\section{CONCLUSION}

In this article, we have established formal relationships between NMR order parameters and conformational entropies, based on a simple dynamical model of internal motions in a protein. Our results demonstrate that caution must be exercised when estimating local conformational entropy from experimental order parameters, as clearly seen from the lack of a one-to-one correspondence between the two quantities. We therefore suggest to use a theoretical framework such as the NCR model in order to relate order parameters to conformational entropies.

\section{ACKNOWLEDGMENTS}

This work has been supported by the European Union (Coordination Action "Focusing NMR on the machinery of life," and the Integrated Infrastructure Initiative "EUNMR"), the Centre National de la Recherche Scientifique (CNRS), and the Agence Nationale de la Recherche (Contract No. ANR-05-BLAN-0255).

${ }^{1}$ V. A. Jarymowicz and M. Stone, Chem. Rev. (Washington, D.C.) 106, 1624 (2006).

${ }^{2}$ A. Mittermaier and L. E. Kay, Science 312, 224 (2006).

${ }^{3}$ A. G. Palmer III and C. Lim, Chem. Rev. (Washington, D.C.) 104, 3623 (2004).

${ }^{4}$ G. Lipari and A. Szabo, J. Am. Chem. Soc. 104, 4546 (1982).

${ }^{5}$ M. Akke, R. Brüschweiler, and A. G. Palmer III, J. Am. Chem. Soc. 115, 9832 (1993).

${ }^{6}$ L. Zidek, M. V. Novotny, and M. J. Stone, Nat. Struct. Biol. 6, 1118 (1999).

${ }^{7}$ D. Abergel and G. Bodenhausen, J. Chem. Phys. 123, 204901 (2005).

${ }^{8}$ D. Abergel andG. Bodenhausen, J. Chem. Phys. 121, 761 (2004).

${ }^{9}$ A. Dhulesia, D. Abergel, and G. Bodenhausen, J. Am. Chem. Soc. 129, 4998 (2007). 
${ }^{10}$ W. T. Coffey, Y. P. Kalmykov, and J. T. Waldron, The Langevin Equation (World Scientific, Singapore, 1996).

${ }^{11}$ V. A. Daragan and K. H. Mayo, J. Phys. Chem. B 103, 6829 (1999).

${ }^{12}$ N. G. van Kampen, Stochastic Processes in Physics and Chemistry (North-Holland, Amsterdam, 1981).

${ }^{13}$ G. Lipari and A. Szabo, J. Am. Chem. Soc. 104, 4559 (1982).

${ }^{14}$ T. I. Igumenova, K. K. Frederick, and A. Joshua Wand, Chem. Rev. (Washington, D.C.) 106, 1672 (2006).
${ }^{15}$ Z. Li, S. Raychaudhuri, and J. A. Wand, Protein Sci. 5, 2645 (2006).

${ }^{16}$ D. Yang and L. E. Kay, J. Mol. Biol. 263, 369 (1996).

${ }^{17}$ J. Kordel, N. J. Skelton, M. Akke, and W. J. Chazin, J. Mol. Biol. 231, 711 (1993).

${ }^{18}$ B. Halle, Proc. Natl. Acad. Sci. U.S.A. 99, 1275 (2002).

${ }^{19}$ N. J. Skelton, J. Kordel, and W. J. Chazin, J. Mol. Biol. 249, 441 (1995).

${ }^{20}$ M. Akke, N. J. Skelton, J. Kordel, A. G. Palmer, and W. J. Chazin, Biochemistry 32, 9832 (1993). 